\title{
G3BP2 is involved in isoproterenol-induced cardiac hypertrophy through activating the NF-KB signaling pathway
}

\author{
Hui-qi HONG ${ }^{1}$, Jing $L^{1}{ }^{1}$, Xiu-li FANG ${ }^{1}$, Yu-hong ZHANG ${ }^{1}$, Yi CAI ${ }^{2}$, Jing YUAN ${ }^{1}$, Pei-qing LIU $^{1}$, Jian-tao YE ${ }^{1,}$ * \\ ${ }^{1}$ Department of Pharmacology and Toxicology, School of Pharmaceutical Sciences, Sun Yat-sen University, Guangzhou 510006, China; \\ ${ }^{2}$ Guangzhou Research Institute of Snake Venom, Guangzhou Medical University, Guangzhou 510182, China
}

\begin{abstract}
The RasGAP SH3 domain-binding proteins (G3BPs) are a family of RNA-binding proteins that can co-ordinate signal transduction and post-transcriptional gene regulation. G3BPs have been shown to be involved in mediating a great diversity of cellular processes such as cell survival, growth, proliferation and apoptosis. But the potential roles of G3BPs in the pathogenesis and progression of cardiovascular diseases remain to be clarified. In the present study, we provide the first evidence that suggests the participation of G3BP2 in cardiac hypertrophy. In cultured neonatal rat cardiomyocytes (NRCMs), treatment with isoproterenol (ISO, 0.1-100 $\mu$ mol/L) significantly elevated the mRNA and protein levels of G3BP2. Similar results were observed in the hearts of rats subjected to 7D-injection of ISO, accompanied by obvious heart hypertrophy and elevated the expression of hypertrophy marker genes ANF, BNP and $\beta-\mathrm{MHC}$ in heart tissues. Overexpression of G3BP2 in NRCMs led to hypertrophic responses evidenced by increased cellular surface area and the expression of hypertrophy marker genes, whereas knockdown of G3BP2 significantly attenuated ISO-induced hypertrophy of NRCMs. We further showed that G3BP2 directly interacted with IkB $\alpha$ and promoted the aggregation of the NF-kB subunit p65 in the nucleus and increased NF-kB-dependent transcriptional activity. NF-KB inhibition with PDTC (50 $\mu$ mol/L) or p65 knockdown significantly decreased the hypertrophic responses in NRCMs induced by ISO or G3BP2 overexpression. These results give new insight into the functions of G3BP2 and may help further elucidate the molecular mechanisms underlying cardiac hypertrophy.
\end{abstract}

Keywords: G3BP2; cardiac hypertrophy; neonatal rat cardiomyocytes; isoproterenol; PDTC; NF-KB; IKB $\alpha$

Acta Pharmacologica Sinica (2018) 39: 184-194; doi: 10.1038/aps.2017.58; published online 17 Aug 2017

\section{Introduction}

Cardiac hypertrophy occurs as a response of the heart to various stresses and is characterized by the enlargement of cardiomyocytes, an increase in protein synthesis, and the reactivation of fetal genes. Hypertrophy may initially be adaptive and help preserve systolic function, but sustained pathological hypertrophy will lead to a decompensated phase with deleterious consequences ${ }^{[1,2]}$. Epidemiological studies have revealed that cardiac hypertrophy is an independent risk factor for cardiac dysfunction and sudden death ${ }^{[3-5]}$. However, despite the great strides that have been made over the past decades in elucidating the pathogenesis of cardiac hypertrophy, the signaling pathways associated with this complex clinical syndrome are far from fully understood.

The RasGAP SH3 domain binding proteins (G3BPs) are a

\footnotetext{
${ }^{*}$ To whom correspondence should be addressed.

E-mail yej@@mail.sysu.edu.cn

Received 2017-01-05 Accepted 2017-04-13
}

highly conserved family of multi-functional RNA binding proteins. In recent years, accumulating evidence has indicated that G3BPs play predominant roles in controlling critical cellular processes that include growth, proliferation and apoptosis ${ }^{[6-8]}$. The G3BP family includes two members in mammals, ie, G3BP1 and G3BP2. G3BP1 knockout mice exhibit growth retardation, embryonic lethality and increased apoptotic cell death ${ }^{[9,10]}$. In contrast, the overexpression of G3BP1 has been shown to induce S-phase entry with elevated cell proliferation ${ }^{[11,12]}$. The G3BP1 protein is upregulated in a diversity of tumors and may present a novel target for cancer therapy ${ }^{[13,14]}$. Thus far, the mechanisms by which G3BP1 executes its functions remains obscure, but previous studies have implicated G3BP1 in the modulation of Ras signaling, RNA metabolism and the ubiquitin proteasome pathway ${ }^{[6,15,16]}$. As a close relative of G3BP1, G3BP2 has similar domain structures comprising the NTF2-like domain and two RNA-binding motifs. Similar to G3BP1, G3BP2 is ubiquitously expressed and also contributes to the formation of stress granules 
(SGs), which are translationally silent sites for mRNA sorting $^{[16,17]}$. Nevertheless, a subtle distinction between these two G3BP isoforms is found in the number of proline-rich $(\mathrm{PxxP})$ motifs in the central region of the proteins. PxxP motifs represent the minimal $\mathrm{SH} 3$ domain-binding consensus sequences. Because $\mathrm{SH} 3$ domain-containing proteins are pivotal signal transducers, the variability in PxxP regions suggests that $\mathrm{G} 3 \mathrm{BP} 1$ and $\mathrm{G} 3 \mathrm{BP} 2$ may associate with different partners to produce distinct cellular outcomes ${ }^{[6,18]}$. To date, G3BP2 is relatively poorly characterized compared with its counterpart G3BP1, and the role of G3BP2 in the cardiovascular system has not been documented. In this study, we reveal the first evidence that G3BP2 is involved in the cardiac hypertrophy induced by isoproterenol (ISO), which may provide additional insight into the potential functions of G3BP2.

\section{Materials and methods}

\section{Primary cell cultures}

Primary cell cultures of neonatal rat cardiomyocytes (NRCMs) were produced according to the methods of our previous report ${ }^{[19]}$. Cardiomyocytes were isolated and purified from the hearts of 1- to 3-day-old Sprague-Dawley (SD) rats. Next, the cells were seeded into 6-well plates at a density of $1 \times 10^{6}$ cells per well with Dulbecco's modified Eagle's medium (DMEM) supplemented with $10 \%$ fetal bovine serum (FBS) and 0.1 mmol/L 5-bromodeoxyuridine. Before treatment, the cells were serum-starved overnight in DMEM with 1\% FBS. Subsequently, ISO (Sigma, St Louis, MO, USA) was added to the culture medium to a final concentration of $1 \mu \mathrm{mol} / \mathrm{L}$, and the cells were further incubated for the indicated duration. In the control group, the cells were supplemented with the same volume of DMEM without ISO.

\section{Animal model, echocardiography and morphometric measures}

All animal experiments were conducted in accordance with the Guide for the Care and Use of Laboratory Animals published by the US National Institutes of Health (NIH Publication No 85-23, revised 1996). This research was approved by the Research Ethics Committee of Sun Yat-sen University. SD rats (male, weighing 200-240 g, SPF grade, Certification № 44008500012063) were supplied by the Experimental Animal Center of Sun Yat-sen University (Guangzhou, China). The animals were submitted to subcutaneous (sc) injections of ISO $\left(1.5 \mathrm{mg} \cdot \mathrm{kg}^{-1} \cdot \mathrm{d}^{-1}\right.$, for $\left.7 \mathrm{~d}\right)$. The rats in the control group received normal saline (NS). Two-dimensionally guided M-mode echocardiography was conducted using a Technos MPX ultrasound system (Esaote, Genoa, Italy) as previously described ${ }^{[20]}$. After echocardiographic assessment, the rats were sacrificed via exposure to $\mathrm{CO}_{2}$. The hearts were rapidly excised. The heart weight (HW) and left ventricle weight (LVW) are expressed as the ratios relative to body weight (BW), and the HW to tibia length (TL) ratio was also calculated. For morphometric measurements, histological cross sections (5 $\mu \mathrm{m}$ thick) from perfuse-fixed hearts were treated with hematoxylin-eosin (HE) staining.
RNA isolation and quantitative real-time polymerase chain reaction ( $q R T-P C R$ )

Total RNA was extracted from cultured NRCMs or rat cardiac tissues with Trizol reagent (Invitrogen, Carlsbad, CA, USA) in accordance to the manufacturer's instructions. Afterwards, 1 $\mu \mathrm{g}$ of total RNA was quantitatively and reversely transcribed to first strand cDNA using Two-step RT kit (Thermo Fisher Scientific, Rockford, IL, USA). Polymerase chain reaction analyses were used to determine the mRNA levels of the target genes with SYBR-Green Quantitative PCR kits (Toyobo Life Science, Osaka, Japan) and an iCycler iQ system (Bio-Rad, Hercules, CA, USA). Rat-specific primers for G3BP2, atrial natriuretic factor (ANF), brain natriuretic polypeptide (BNP), myosin heavy chain $\beta$ ( $\beta$-MHC), and glyceraldehyde-3-phosphate dehydrogenase (GAPDH) were synthesized by Sangon Biotech (Shanghai, China) as listed in Supplementary Material Table S1. GAPDH served as an endogenous control.

\section{Western blot and co-immunoprecipitation (co-IP)}

The primary antibodies against G3BP1 (diluted 1:2000) and G3BP2 (diluted 1:2000) were produced by Sigma (St Louis, MO, USA). The primary antibodies against ANF (diluted 1:200), BNP (diluted 1:1000), $\beta$-MHC (diluted 1:10000), p65 (diluted 1:1000), p-p65 (diluted 1:1000) and IкBa (diluted 1:1000) were purchased from Cell Signaling Technology (Beverly, MA, USA). The antibodies against GAPDH (diluted 1:5000) and Lamin B1 (diluted 1:1000) were from Santa Cruz Biotechnology (Santa Cruz, CA, USA). Nuclear protein was isolated using CelLytic NuCLEAR Extraction Kits (Sigma, St Louis, MO, USA). Western blot analyses were performed following previously described protocol ${ }^{[21]}$. The protein band intensities were measured with LabWorks software (Bio-Rad, Hercules, CA, USA). For the co-IP assays, $200 \mu \mathrm{g}$ of total protein was incubated overnight with $1 \mu \mathrm{g}$ anti-IKBa antibody and further treated with protein G-agarose beads (Pierce, Rockford, IL, USA) for $4 \mathrm{~h}$ at $4{ }^{\circ} \mathrm{C}$. The immunoprecipitates were detected by Western blot.

\section{Plasmid transfection and RNA interference}

The NRCMs were transiently transfected with FLAG-tagged G3BP2 or an empty vector using Lipofectamine 2000 (Invitrogen, Carlsbad, CA, USA) according to the manufacturer's instructions. Three different small interference RNAs (siRNAs) for G3BP2 and negative control siRNAs were purchased from GenePharma (Shanghai, China). The sequences are provided in Supplementary Material Table S2. RNA interference was conducted using Lipofectamine 2000. At $48 \mathrm{~h}$ after transfection, the cells were harvested for RNA or protein extraction to compare the silencing efficacies (Figure S1).

\section{Measurement of the cell surface area}

NRCMs in 48-well plates were fixed with $4 \%$ paraformaldehyde for $15 \mathrm{~min}$ at room temperature. The cells were further incubated for $30 \mathrm{~min}$ with $0.1 \%$ rhodamine-phalloidin (Invitrogen, Carlsbad, CA, USA) and washed three times with phosphatebuffered saline (PBS). The coverslips were mounted in Prolong 
Gold anti-fade reagent with 4', 6-diamidino-2-phenylindole (DAPI, Cell Signaling Technology, MA, USA) and inspected with a High Content Screening system (Thermo Fisher Scientific, Rockford, IL, USA). Cells from randomly selected fields (50 for each group) were examined, and the surface areas were measured using built-in image analysis software.

\section{Immunofluorescence (IF) assay}

The NRCMs grown on coverslips were fixed in $4 \%$ paraformaldehyde and permeabilized using $0.3 \%$ Triton X-100. After three washes with PBS, the cells were blocked with normal goat serum at room temperature for $1 \mathrm{~h}$. Primary p65 antibody (diluted 1:50, Cell Signaling Technology, MA, USA) was applied for $1 \mathrm{~h}$ and followed by incubation with Alexa Fluor 488-conjugated secondary antibody (diluted 1:500, Santa Cruz Biotechnology, CA, USA). The coverslips were mounted with DAPI. The images were captured with a confocal microscope (Zeiss, Oberkochen, Germany).

\section{Dual-luciferase reporter gene assay}

The NRCMs were seeded into 96-well plates at a density of $5 \times 10^{4}$ cells per well and then co-transfected with NF-kB luciferase reporter plasmid pGL4.32 (containing the p65 DNA binding sequence GGGACTTTCC, $100 \mathrm{ng}$ per well) and pRL-TK internal control vector (20 ng per well). After $6 \mathrm{~h}$ of incubation, the cells were serum-deprived for $12 \mathrm{~h}$ and submitted to ISO treatment, RNA interference, or FLAG-G3BP2 transfection. The cells were harvested and lysed in passive lysis buffer. The luciferase activity was measured with a dualluciferase reporter assay system (Promega, Fitchburg, MA, USA), and the activity was normalized to the Renilla luciferase activity of the pRL-TK.

\section{Statistical analysis}

The data are presented as the mean \pm the standard errors of the mean (SEM). The differences between two groups were analyzed with unpaired Student's $t$-tests. The statistical analyses among multiple groups were performed with one-way ANOVAs with Tukey's post hoc tests. In all cases, the differences were considered statistically significant at $P<0.05$.

\section{Results \\ G3BP2 expression in neonatal rat cardiomyocytes treated with isoproterenol}

ISO, a nonselective $\beta$-adrenergic receptor ( $\beta$-AR) agonist, has been widely used to induce cardiac hypertrophy ${ }^{[22,23]}$. Our data revealed that the mRNA expression of G3BP2 in cardiomyocytes was elevated in a dose- and time-dependent manner following ISO treatment (Figure 1A, 1B). ISO stimulation also led to an evident increase in the G3BP2 protein level (Figure 1C, 1D).

\section{G3BP2 expression in ISO-induced cardiac hypertrophy in rats}

To further investigate the influence of ISO on G3BP2 expression in vivo, SD rats were subjected to ISO injections (1.5 $\left.\mathrm{mg} \mathrm{kg}^{-1} \cdot \mathrm{d}^{-1}, \mathrm{sc}\right)$ for $7 \mathrm{~d}$. The hearts of the ISO-treated rats were enlarged compared with those from the control group that was treated with normal saline (NS) and exhibited representative hypertrophic pathological changes as shown by HE staining (Figure 2A-C). Echocardiography indicated that the left ventricular anterior wall thickness (LVAW), left ventricular posterior wall thickness (LVPW), fractional shortening (FS) and ejection fraction (EF) were increased, whereas the left ventricular internal diameter (LVID) was decreased in the ISO group (Figure 2D and Supplementary Material Table S3). Additionally, the heart weight-to-body weight (HW/BW) ratio and the left ventricle weight-to-body weight (LVW/BW) ratio were both increased after ISO treatment (Figure 2E). The heart weight/tibia length (HW/TL) ratio was also elevated following ISO treatment (Supplementary Material Figure S1). Additionally, the protein levels of hypertrophic markers, including $\mathrm{ANF}, \mathrm{BNP}$ and $\beta-\mathrm{MHC}$, were notably upregulated (Figure $2 \mathrm{G})$. The above results demonstrated that the rat model of cardiac hypertrophy was successfully established. Furthermore, the qRT-PCR and Western blot analyses demonstrated that the expression of G3BP2 was elevated in the cardiac tissues of the ISO-injected rats (Figure 2F and 2G).

\section{G3BP2 knockdown attenuated ISO-stimulated hypertrophy in neonatal rat cardiomyocytes}

In this study, ISO treatment resulted in a remarkable upregulation of G3BP2 expression in cultured cardiomyocytes and in rat heart tissues. Subsequently, we employed the approach of RNA interference to explore the role of G3BP2 in hypertrophy stimulated by ISO. Cultured cardiomyocytes were transfected with three different siRNAs termed si-1, si-2, and si-3. Among these siRNAs, si-2 was the most effective for G3BP2 knockdown (Supplementary Material Figure S2). The cell surface areas and levels of ANF, BNP and $\beta$-MHC were measured (Figure $3 \mathrm{~A}-3 \mathrm{C}$ ). The results revealed that the downregulation of G3BP2 with si-2 (siG3BP2) blocked the increase in cell surface area and the expressions of hypertrophic biomarkers triggered by ISO incubation $(1 \mu \mathrm{mol} / \mathrm{L}$ for $12 \mathrm{~h})$. In contrast, the overexpression of G3BP2 led to obvious hypertrophic responses in the NRCMs as implied by enlarged cell surface areas and increased levels of hypertrophic biomarkers (Figure 3D-3F).

NF-kB inhibition ameliorated the cardiomyocyte hypertrophy induced by ISO or G3BP2 overexpression

NF-kB activation provokes the hypertrophic growth of cardiomyocytes $^{[24,25]}$. To investigate whether NF-KB signaling is involved in the pro-hypertrophic effects of G3BP2, pyrrolidine dithiocarbamate (PDTC), which is a well-established inhibitor of NF-KB activity, was added to the NRCMs at a final concentration of $50 \mu \mathrm{mol} / \mathrm{L}^{[26,27]}$. As illustrated in Figure $4 \mathrm{~A}-4 \mathrm{C}$, both ISO simulation and G3BP2 overexpression led to obvious increases in cell surface area and the expressions of ANF, BNP and $\beta$-MHC, and these increases were suppressed by PDTC. Additionally, PDTC attenuated the nuclear aggregation of p65 and inhibited the NF-kB-dependent transcriptional activity induced by ISO or G3BP2 overexpression (Figure 4D-4F). Moreover, the elevations of the mRNA levels of the hypertro- 
A

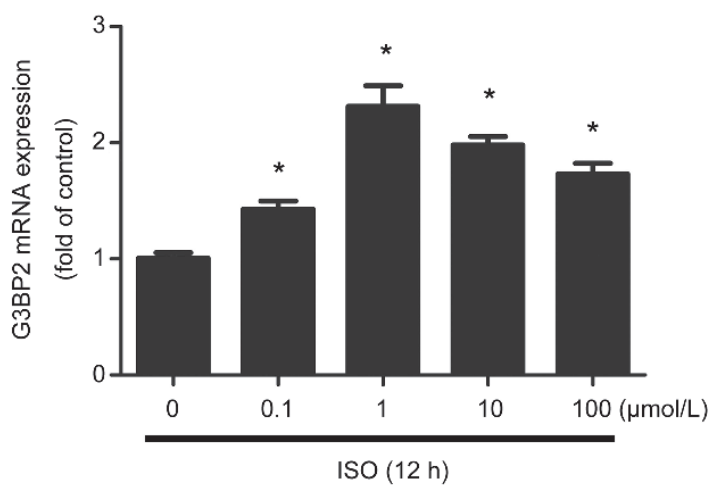

C
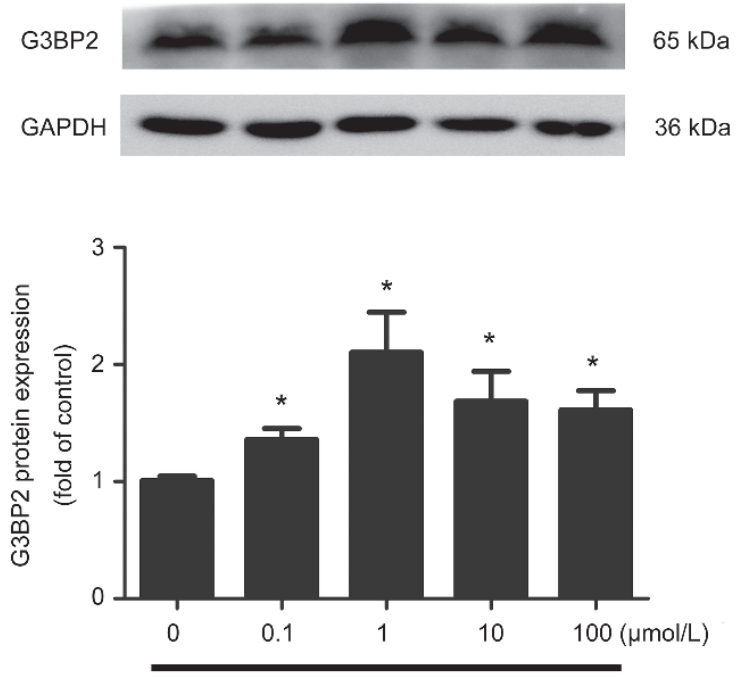

ISO (12 h)
B

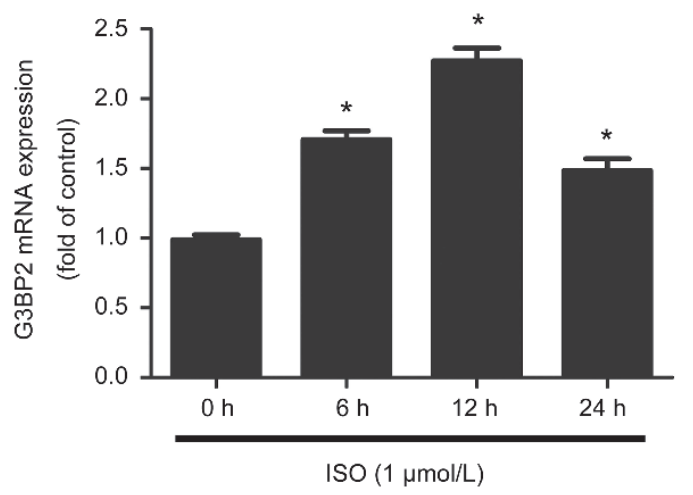

D
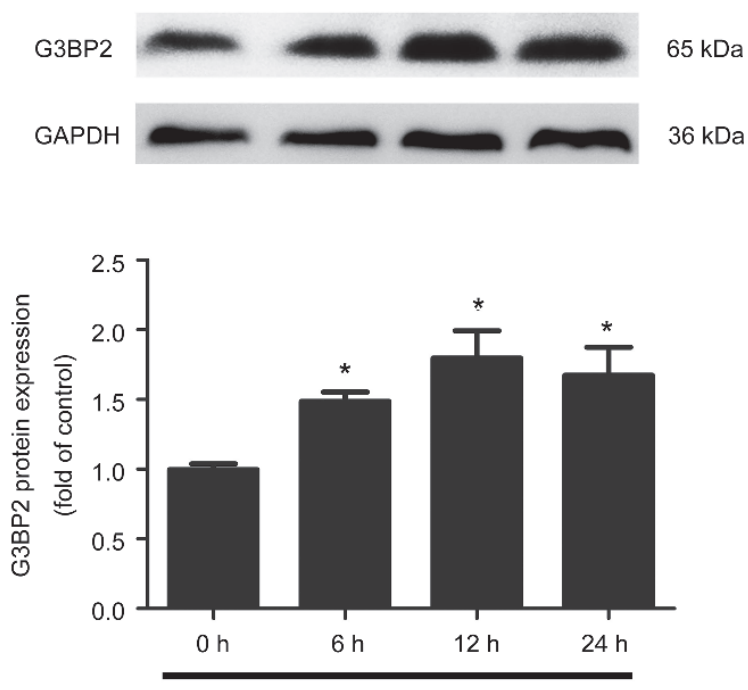

ISO (1 $\mu \mathrm{mol} / \mathrm{L})$

Figure 1. G3BP2 expression was increased in neonatal rat cardiomyocytes following ISO treatment. NRCMs were incubated with various concentrations of ISO for $12 \mathrm{~h}$ or with $1 \mu \mathrm{mol} / \mathrm{L}$ ISO for the indicated durations. (A and B) The mRNA levels of G3BP2 were determined by qRT-PCR. (C and D) The protein expression of G3BP2 was measured by Western blot. The results were normalized (by GAPDH) and are presented as the fold-changes relative to the control levels. The data are presented as the mean \pm SEM. ${ }^{*} P<0.05$ vs control. $n=3$.

phic marker genes were ameliorated by p65 knockdown with siRNA (Figure 4G).

G3BP2 interacted with $І \kappa B \alpha$ and promoted the nuclear localization of NF-KB subunit $\mathrm{p} 65$ in cardiomyocytes

A previous study showed that in HeLa cells, G3BP2 can combine with $\mathrm{I} \kappa \mathrm{Ba}$, which presumably contributes to the activation of the NF- $\mathrm{B}$ signal transduction cascade ${ }^{[28]}$. Consistently, the immunoprecipitation assay revealed a direct interaction between IKBa and G3BP2 in the NRCMs, and this interaction was strengthened by ISO treatment (Figure 5A). Moreover, the Western blot results showed that ISO simulation facilitated the nuclear aggregation of NF-kB subunit p65, and this facilitation was partially inhibited by transfection with G3BP2 siRNA (Figure 5B). The intracellular distribution of p65 in cardiomyocytes was also authenticated with IF assays. As indicated by Figure 5C, p65 was distributed in both the cytoplasm and the nucleus without stimulation. ISO induced its nuclear translocation, and this process was attenuated by G3BP2 knockdown. In contrast, the overexpression of G3BP2 increased the localization of p65 in the nucleus (Figure 5D, 5E).

Subsequently, the effects of G3BP2 on NF-kB-related transcriptional activity were evaluated with a dual luciferase reporter gene assay. G3BP2 overexpression gave rise to NF-kB-Luc reporter gene activity that was similar to the effect of ISO treatment. In contrast, the knockdown of G3BP2 repressed the up-regulation of NF-kB-Luc reporter gene activity (Figure 5F and 5G). Taken together, these findings suggested that G3BP2 might participate in ISO-induced cardiomyocyte hypertrophy by enhancing NF-kB signaling.

\section{Discussion}

The G3BP family of proteins has been postulated to link signal transduction with RNA metabolism to maintain 
A

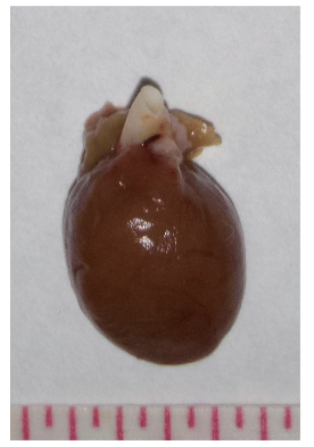

B

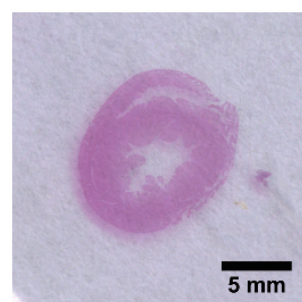

C

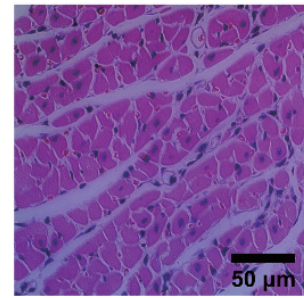

D

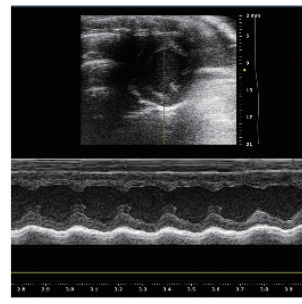

NS
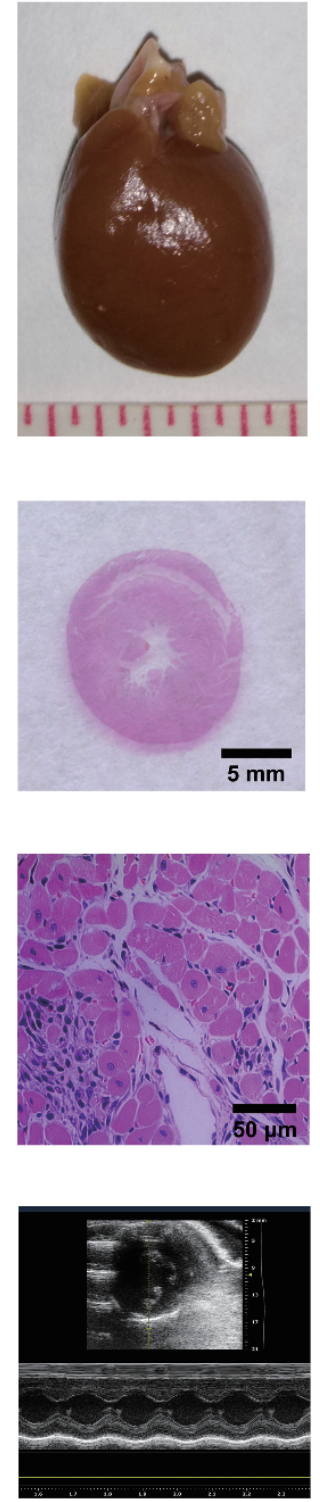

ISO
E

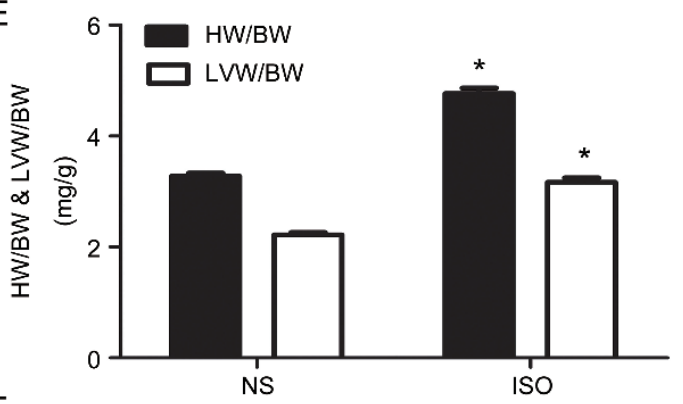

F

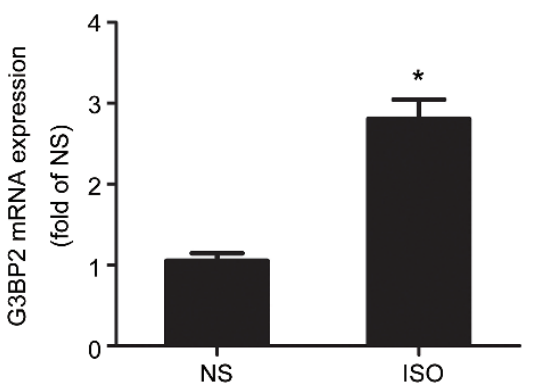

G
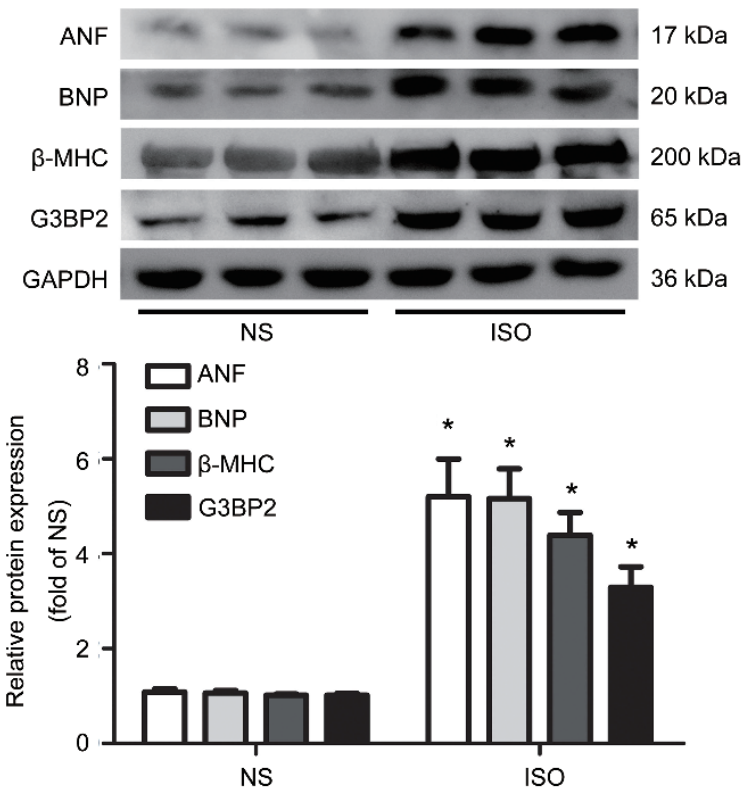

Figure 2. ISO treatment resulted in cardiac hypertrophy accompanied by elevated G3BP2 expression in SD rats. SD rats were subcutaneously injected with ISO $\left(1.5 \mathrm{mg}^{-1} \mathrm{~kg}^{-1} \mathrm{~d}^{-1}\right)$ for $7 \mathrm{~d}$. The control animals received normal saline (NS). (A-C) Pathologic changes in the hearts were observed by H\&E staining and (D) echocardiography. (E) The HW/BW and LVW/BW ratios were calculated. (F) G3BP2 mRNA expression in heart tissues was detected by qRT-PCR. (G) The protein levels of G3BP2 and hypertrophic markers, including ANF, BNP and $\beta$-MHC, were measured by Western blot. The data are presented as the mean \pm SEM. ${ }^{*} P<0.05$ vs NS group. $n=8$.

cell survival and homeostasis ${ }^{[7]}$. In cardiomyocytes, it has been demonstrated that G3BP1 binds to and stabilizes the mRNA encoding Cdk7, which leads to elevated Cdk7 protein expression and cell growth ${ }^{[29]}$. Additionally, G3BP1 mediates the decline of the mature miRNA- 1 level, which is necessary for the increase in protein synthesis during cardiac hypertrophy ${ }^{[30]}$. However, the potential functions of G3BP2 in the cardiovascular system remain to be determined. Here, we observed that G3BP2 expression was increased in ISO- treated neonatal rat cardiomyocytes (Figure 1). Upregulations of G3BP2 mRNA and protein levels were also identified in the cardiac tissues of SD rats that received ISO injections (Figure 2). Furthermore, the overexpression of G3BP2 led to cardiomyocyte hypertrophy, but G3BP2 RNA interference significantly attenuated the hypertrophic responses stimulated by ISO (Figure 3). To our knowledge, these data provide previously unreported evidence indicating the participation of G3BP2 in ISO-induced cardiac hypertrophy. 
A
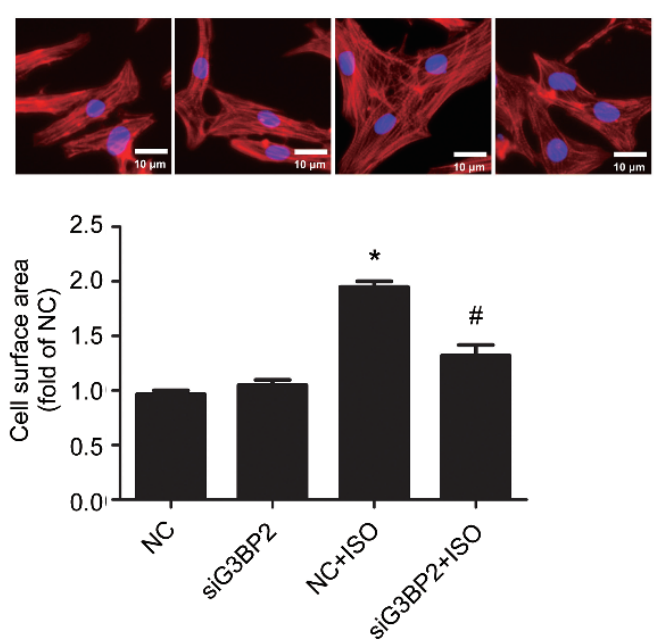

B

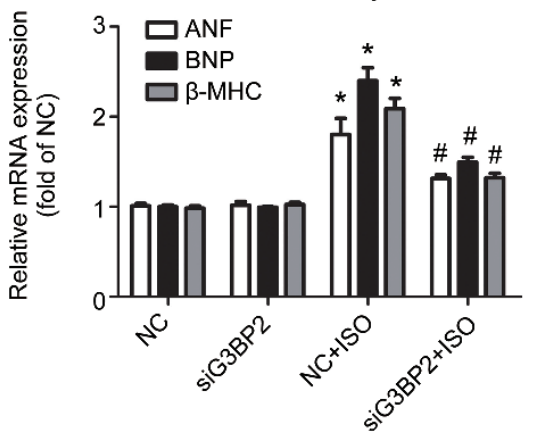

C
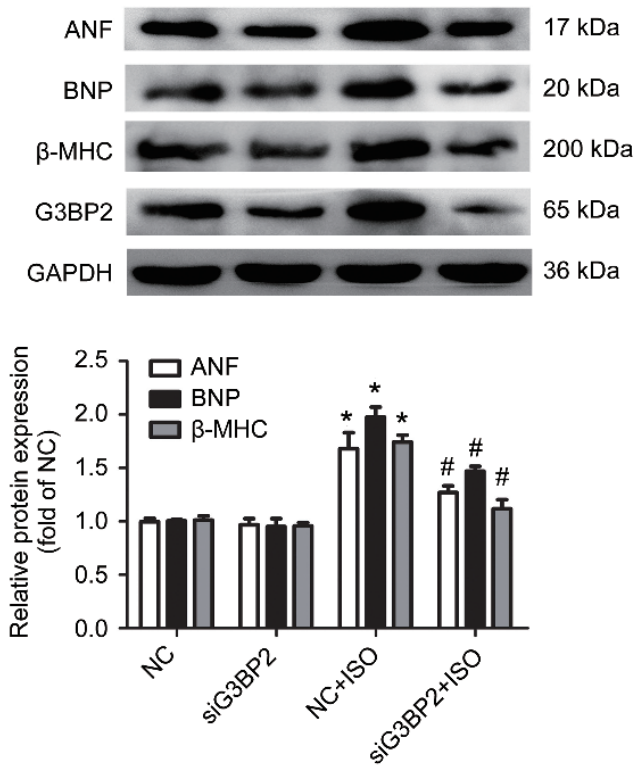

D

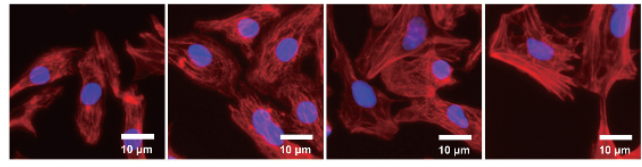

E
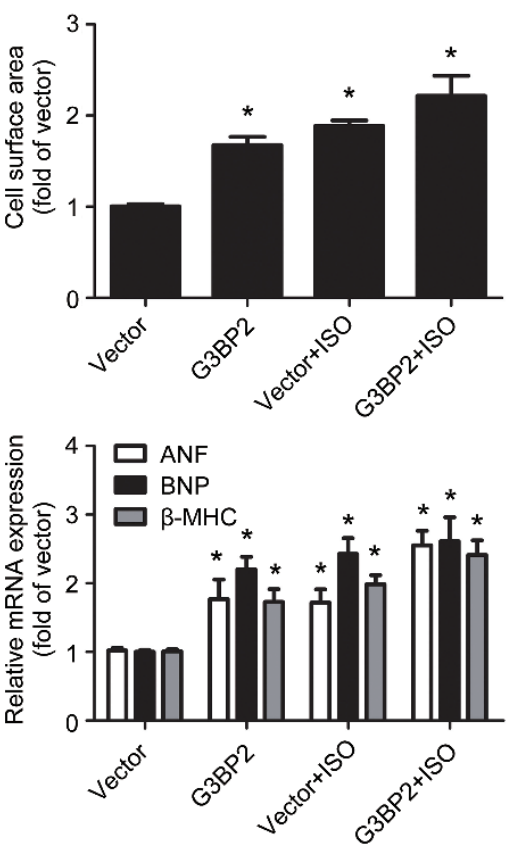

F
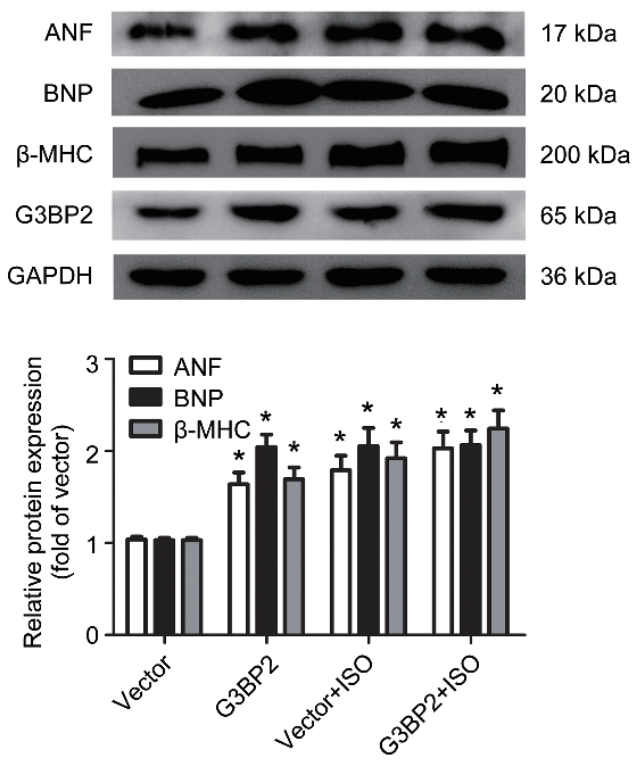

Figure 3. G3BP2 knockdown attenuated ISO-induced hypertrophy, and G3BP2 overexpression led to hypertrophic responses in cardiomyocytes. NRCMs were treated with G3BP2 siRNA (si-G3BP2) or negative control (NC) for $48 \mathrm{~h}$ and further incubated with or without $1 \mu \mathrm{mol} / \mathrm{L}$ ISO for $12 \mathrm{~h}$. (A) The cell surface area was measured with rhodamine-phalloidin staining using a $200 \times$ objective. (B and C) The mRNA and protein levels of ANF, BNP and B-MHC were detected by qRT-PCR and Western blot, respectively. (D-F) The NRCMs were transfected with G3BP2 plasmid or empty vector for $24 \mathrm{~h}$ and subsequently treated with $1 \mu \mathrm{mol} / \mathrm{L}$ ISO for $12 \mathrm{~h}$. The hypertrophic responses of the cells were determined by measuring the cell surface area and the expressions of the hypertrophic markers. Mean \pm SEM. ${ }^{*} P<0.05$ vs NC or vector group. ${ }^{\#} P<0.05$ vs NC plus ISO group. $n=3$.

G3BP1 and G3BP2 are encoded by distinct genes on chromosomes. Structural analysis has revealed that their protein sequences are $60 \%$ identical ${ }^{[31]}$. Despite this high homology at the protein level, recent research has shown that the func- 
A
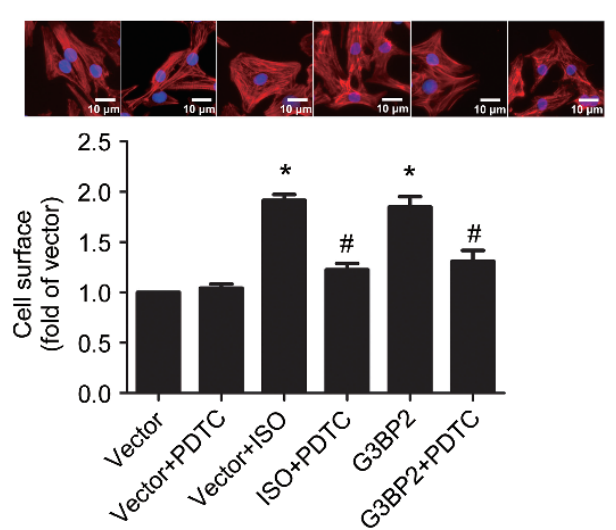

B

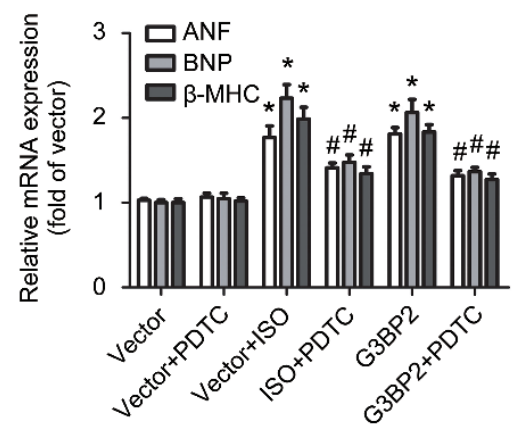

C

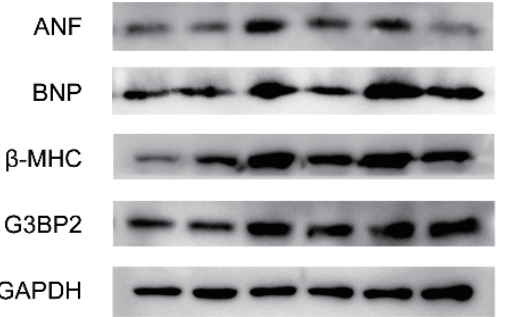

$17 \mathrm{kDa}$

$20 \mathrm{kDa}$

$200 \mathrm{kDa}$

$65 \mathrm{kDa}$

$36 \mathrm{kDa}$

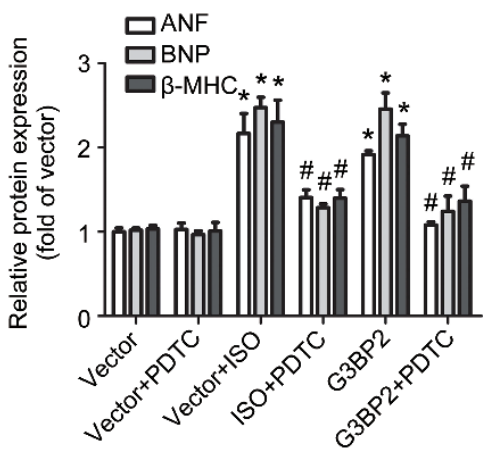

G

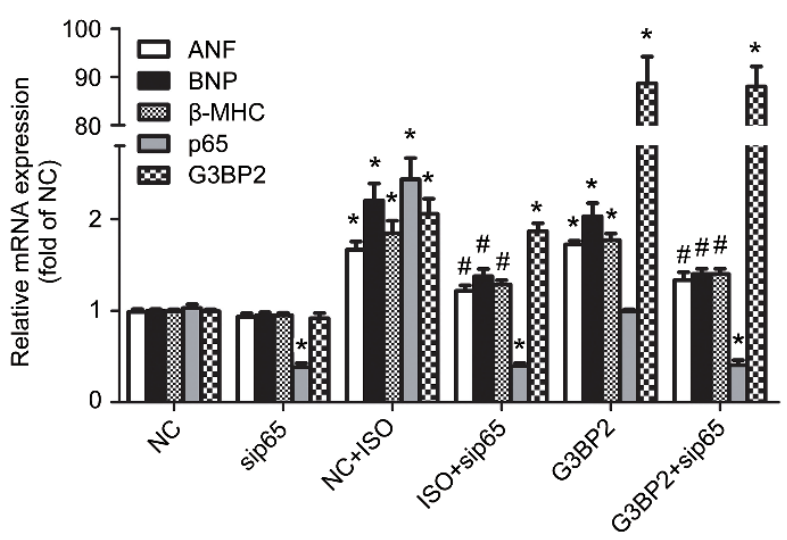

D

$E$

$\mathrm{F}$
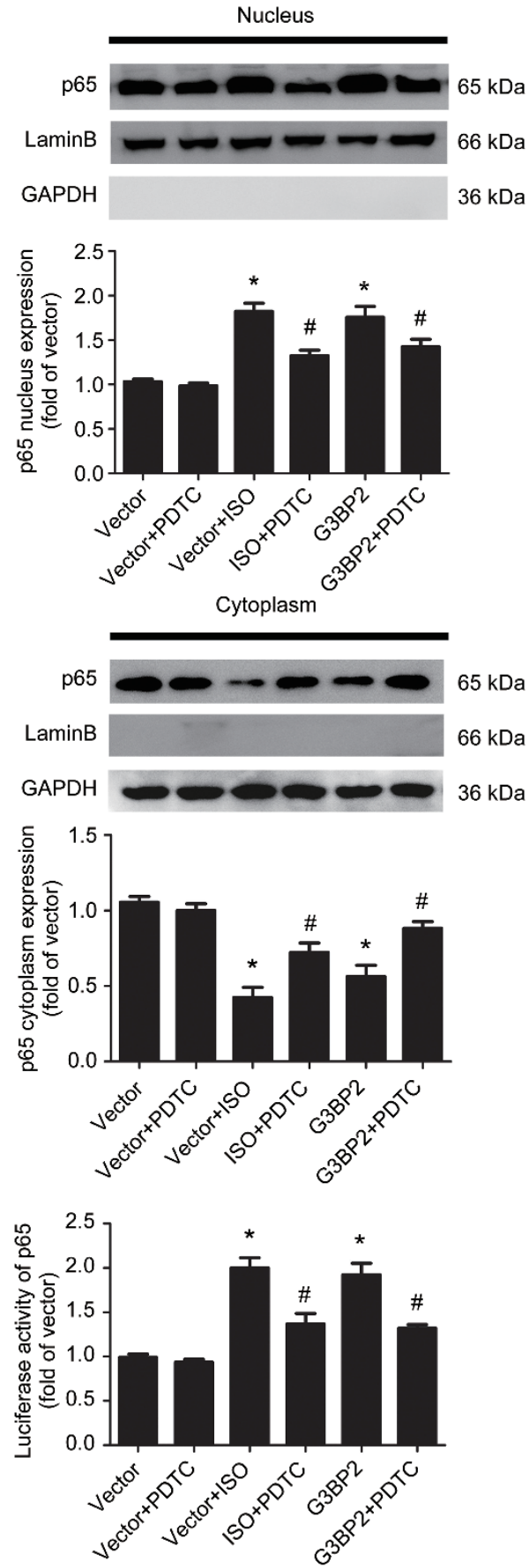

Figure 4. The inhibition of NF-KB with both PDTC and RNA interference ameliorated the cardiomyocyte hypertrophy triggered by ISO or G3BP2 overexpression. (A) NRCMs were pre-incubated with $50 \mu \mathrm{mol} / \mathrm{L}$ PDTC for $1 \mathrm{~h}$ and then subjected to ISO treatment ( $1 \mu \mathrm{mol} / \mathrm{L}$ for $12 \mathrm{~h}$ ) or G3BP2 plasmid transfection. The cell surface area was measured with rhodamine-phalloidin staining. $\times 200$. (B, C) The mRNA and protein levels of ANF, BNP and B-MHC. (D, E) The levels of p65 in the nucleus and cytoplasm. (F) NF-kB-dependent transcriptional activity was tested with a dual-luciferase reporter gene assay. (G) NRCMs were treated with p65 siRNA (si-p65) or negative control (NC) for $48 \mathrm{~h}$ and then subjected to ISO treatment or G3BP2 overexpression. The mRNA levels of ANF, BNP and $\beta$-MHC were detected. Mean \pm SEM. ${ }^{*} P<0.05$ vs vector or NC group. ${ }^{\#} P<0.05$ vs vector plus ISO, NC plus ISO, or G3BP2 overexpression group. $n=3$. 
tions of the G3BPs do not fully overlap. For example, G3BP1 exhibits phosphorylation-dependent endoribonuclease activity ${ }^{[7,8]}$, which has not been reported for G3BP2. Moreover, Wnt-induced gene transcription is attenuated by G3BP2 knockdown, and this effect cannot be rescued by G3BP1 overexpression ${ }^{[32,33]}$. In the present study, we found that following ISO treatment, the mRNA and protein levels of G3BP2 were obviously elevated in the NRCMs, whereas G3BP1 expression remained intact (Supplementary Material Figure S2). Together with previous reports, these findings may elucidate the different functions that are attributed to the G3BP isoforms.

$\beta$-adrenergic receptors ( $\beta$-ARs) are critical for the sympathetic modulation of cardiac performance ${ }^{[34]}$. Treatment with ISO, which is a nonselective agonist of $\beta$-ARs, can induce cardiac hypertrophy and subsequent heart failure in experimental animals ${ }^{[22,23]}$. Our data revealed that ISO stimulation enhanced G3BP2 expression both in vitro and in vivo in addition to the development of hypertrophy (Figure 1 and Figure 2E and F), but the underlying mechanisms remain unclear. G3BP is an inducible gene product of human epidermal growth factor receptor 2 (HER2) signaling. G3BP expression is elevated in cells in parallel with the overexpression of HER2 ${ }^{[35]}$. A recent study showed that in response to $\beta_{2}$-AR stimulation by ISO, STAT3 is activated and translocates to the nucleus where it binds to the promoter of HER2 and subsequently upregulates HER2 expression ${ }^{[36]}$. These findings suggest the possibility that ISO may manipulate G3BP2 expression by modulating STAT3-dependent HER2 expression via $\beta_{2}$-AR. Further investigations are certainly required to clarify this issue.

Mounting evidence has confirmed a prominent relationship between the NF-KB signaling pathway and cardiac hypertrophy. The over activation of NF-kB has been observed in hypertrophic and failing human hearts, whereas the inhibition of NF-KB can attenuate cardiac hypertrophy ${ }^{[37,38]}$. Moreover, it has been demonstrated that the activation of NF-KB is required for ISO-induced cardiac hypertrophy ${ }^{[39]}$. In line with previous studies, we observed that ISO promoted the aggregation of p65 in the nucleus and enhanced NF-kB transcriptional activity in cultured cardiomyocytes. Similar results were also observed in G3BP2-overexpressing cells (Figure 4D-F). Treatment with the NF-kB inhibitor PDTC attenuated the hypertrophic responses and simultaneously suppressed the nuclear localization and transcriptional activity of NF- $\mathrm{kB}$ that was stimulated by ISO or G3BP2 overexpression (Figure 4). These findings suggest that G3BP2 may participate in the development of hypertrophy in a NF-kB-dependent manner.

Although PDTC is a well-established inhibitor of NF- $\mathrm{KB}$ activation that acts via preventing the phosphorylation and degradation of IKBa ${ }^{[40,41]}$, some studies have shown that it may lead to off-target effects. As a chelating agent and antioxidant, PDTC induces glutathione synthesis at a concentration lower than that needed to inhibit the biological actions of NF- $\mathrm{KB}^{[42]}$. Additionally, PDTC can promote stromelysin expression via the stimulation of the tyrosine kinase-AP-1 pathway in a manner that is independent of its suppression of NF-kB ${ }^{[43]}$. Thus, we performed further experiments using p65 knockdown by
siRNA. The results revealed that both ISO and G3BP2 overexpression-induced expression of hypertrophic marker genes were attenuated following the depletion of p65 (Figure 4G), which further indicates the involvement of NF- $\mathrm{KB}$ signaling in G3BP2-regulated hypertrophy.

In response to stimulation, IKBa is phosphorylated and subsequently degraded through the ubiquitin-proteasome proteolytic pathway. This process releases p65 to enter the nucleus and then activate the expression of a diversity of genes that are associated with cell survival and growth ${ }^{[4]}$. Additionally, unregulated ІкBa translocates into the nucleus, dissociates p65 from DNA, and re-exports it back to the cytoplasm ${ }^{[45,46]}$. The $\mathrm{N}$-terminal region of IкBa contains a short cytoplasmic retention sequence (CRS) that can be recognized by G3BP2. G3BP2 has been shown to interact directly with IKBa, and the overexpression of G3BP2 retains IKBa in the cytoplasm ${ }^{[28]}$. Additionally, the IKBa-interacting domain of G3BP2 has been mapped, and the central acid-rich region of G3BP2 has been indicated to be sufficient for its binding with ІкBa and the promotion of cytoplasmic retention ${ }^{[28]}$. These facts suggest a potential link between G3BP2 and NF-kB signaling because G3BP2 may manipulate the nucleocytoplasmic distribution of IкBa and thereby influence NF-KB activity. Here, we found that G3BP2 co-immunoprecipitated with IKBa, and the association was enhanced by ISO treatment (Figure 5A). G3BP2 overexpression resulted in increased aggregation of p65 in the nucleus accompanied by elevated NF-kB transcriptional activity. The increased nuclear entry of p65 and NF-kB activation following ISO stimulation was attenuated by G3BP2 knockdown (Figure $5 B$ and $5 C$ ). Although quite preliminary, these results may suggest the possibility that G3BP2 allows NF-KB to remain active in the nucleus presumably by interacting with IкBa and retaining it in the cytoplasm. Intriguingly, we also observed that G3BP2 overexpression facilitated p65 phosphorylation and simultaneously downregulated the protein level of IкBa (Supplementary Material Figure S5), which supports its multiple activation effects on NF-kB signaling. Nevertheless, the underlying mechanisms still require elucidation.

Despite the evidence presented here concerning the potential connection between G3BP2 and cardiac hypertrophy, there are some obvious limitations to our studies. First, the participation of G2BP2 in the pathogenesis of cardiac hypertrophy should be further confirmed in vivo, for example, using rats with cardiac-specific depletion or overexpression of G3BP2. Second, whether the same conclusions can be drawn from the TAC-induced pressure overload hypertrophy animal model is unknown. Additionally, osmotic minipumpbased infusion of ISO is preferable to subcutaneous injection because the former ensures constant and sustained drug delivery. Moreover, our experiments also revealed that G3BP2 expression was increased by ISO in cardiac fibroblasts (Supplementary Material Figure S6). As the most prevalent cell type in the heart, fibroblasts are known to contribute to the development of cardiac remodeling and heart failure ${ }^{[47]}$. Therefore, the potential influence of enforced G3BP2 expression in cardiac fibroblasts should be considered. 
A

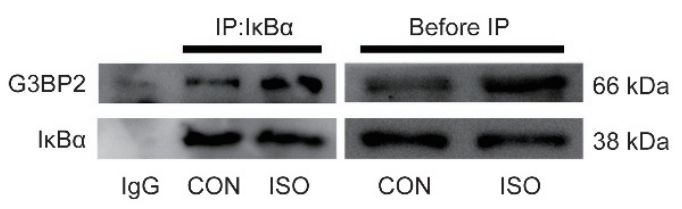

D

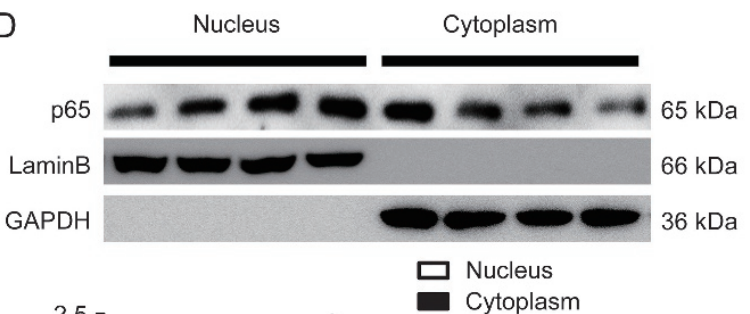

B
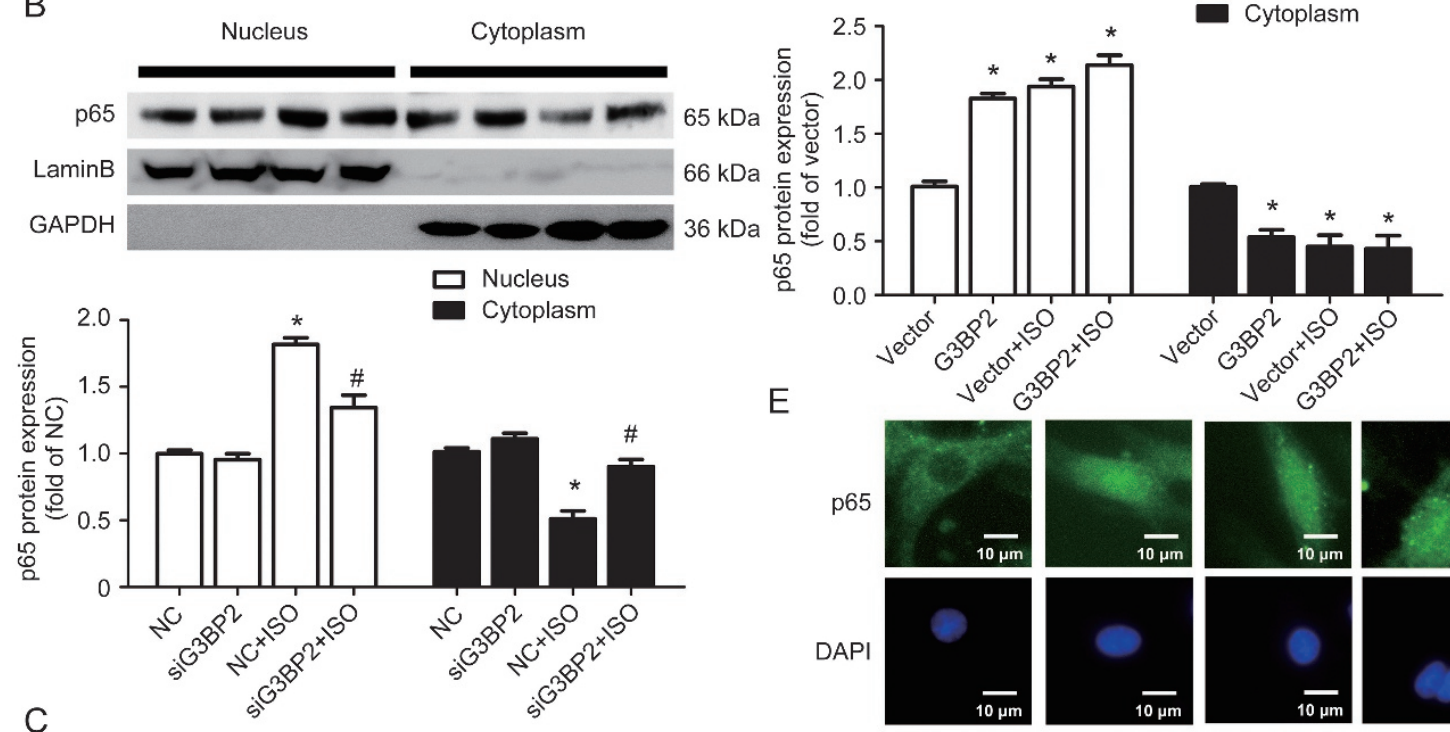

C
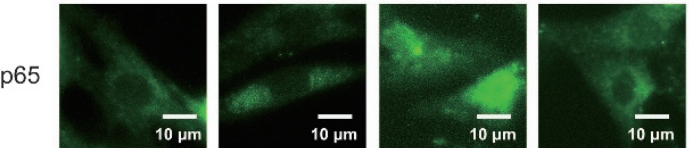

DAPI
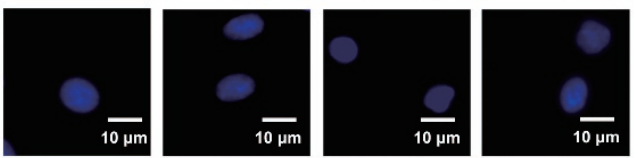
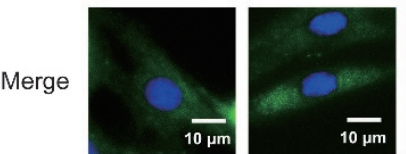

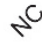

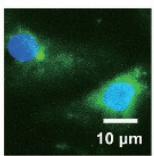

$5^{x^{x^{5}}}$<smiles>[Te]=[Te]</smiles>

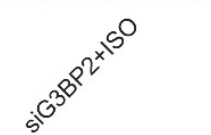

E
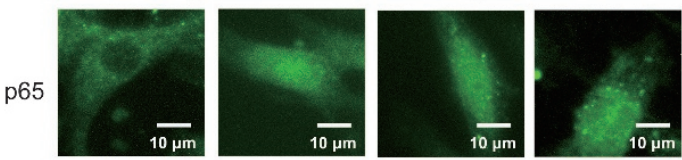

DAPI
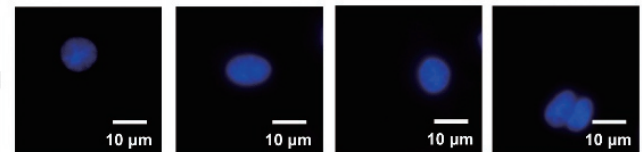

Merge
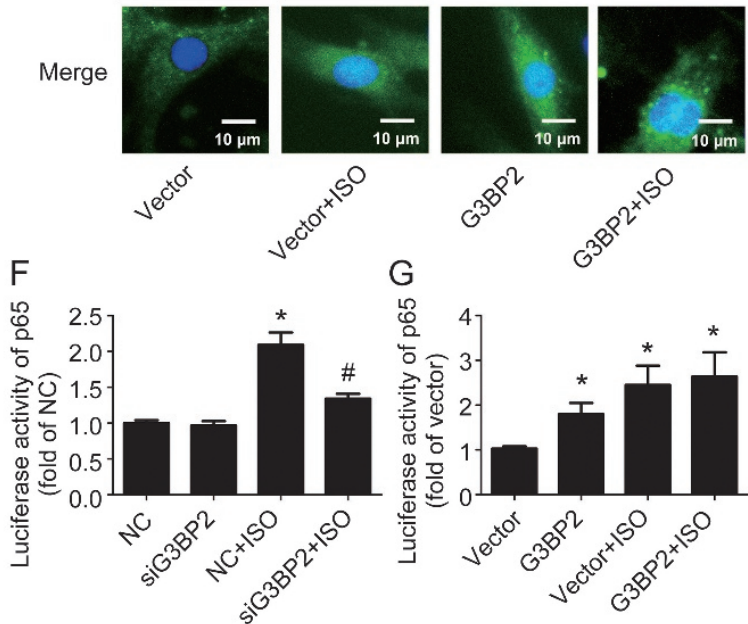

$\mathrm{G}$

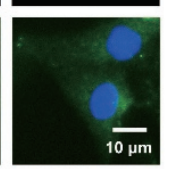

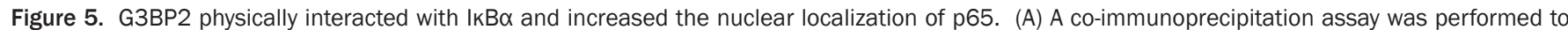

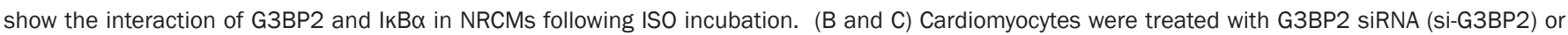

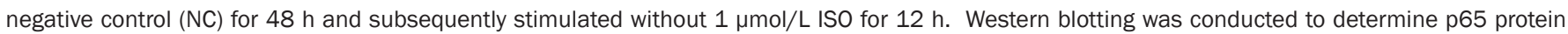

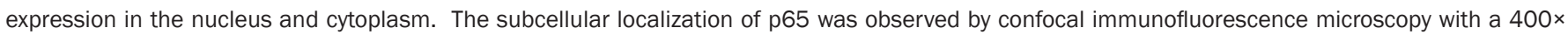

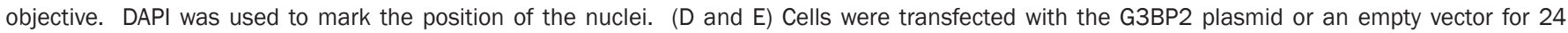

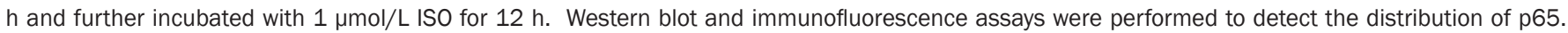

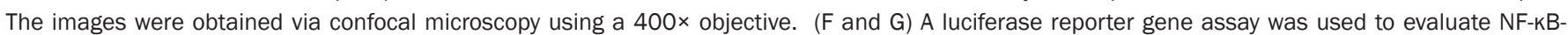
dependent transcriptional activity. The data are presented as the mean \pm SEM. ${ }^{*} P<0.05$ vs NC or vector group. ${ }^{\#} P<0.05$ vs NC plus ISO group. $n=3$.

In summary, our present study revealed that G3BP2 is involved in ISO-induced cardiac hypertrophy. G3BP2 expression was significantly increased in cardiomyocytes following ISO treatment and in the heart tissues of rats subjected to ISO injection. The overexpression of G3BP2 led to hypertrophic responses in cultured cardiomyocytes, and RNA interference with G3BP2 protected the cells from hypertrophy. The pro-hypertrophic effects of G3BP2 could be partially attributed to its positive influence on NF-kB activation. These findings add new information to the limited literature regarding the biological functions of G3BP2 and will help improve the understanding of the molecular mechanisms underlying cardiac 
hypertrophy.

\section{Acknowledgements}

This work was supported by grants from the National Natural Science Foundation of China (№ 81072641, 81273499, $81473205,81400359,81673433$, and 81200096), the Team item of the Natural Science Foundation of Guangdong Province (No S2011030003190), the Major Project of Guangdong Provincial Department of Science and Technology (No 2013B090700010), the Major Project of Platform Construction Education Department of Guangdong Province (No 2014GKPT002), and the Special Program for Applied Science and Technology of Guangdong Province (№ 2015B020232009).

\section{Author contribution}

Hui-qi HONG, Jing LU and Pei-qing LIU designed and conducted the experiments and analyzed the data. Xiu-li FANG, Yu-hong ZHANG, Yi CAI, and Jing YUAN partially performed some experiments. Jian-tao YE designed the experiments, analyzed data, wrote the manuscript, and conceived of the whole project.

\section{Supplementary information}

Supplementary information is available at the website of Acta Pharmacologica Sinica.

\section{References}

1 Heineke J, Molkentin JD. Regulation of cardiac hypertrophy by intracellular signalling pathways. Nat Rev Mol Cell Biol 2006; 7: 589600.

2 Balakumar P, Jagadeesh G. Multifarious molecular signaling cascades of cardiac hypertrophy: can the muddy waters be cleared? Pharm Res 2010; 62: 365-83.

3 Sabbah HN. Silent disease progression in clinically stable heart failure. Eur J Heart Fail 2017; 19: 469-78.

4 Veselka J, Anavekar NS, Charron P. Hypertrophic obstructive cardiomyopathy. Lancet 2017; 389: 1253-67.

5 Michels M, Olivotto I, Asselbergs FW, van der Velden J. Lifelong tailoring of management for patients with hypertrophic cardiomyopathy: awareness and decision-making in changing scenarios. Neth Heart J 2017; 25: 186-99.

6 Irvine K, Stirling R, Hume D, Kennedy D. Rasputin, more promiscuous than ever: a review of G3BP. Int J Develop Biol 2004; 48: 1065-77.

7 Gallouzi IE, Parker F, Chebli K, Maurier F, Labourier E, Barlat I, et al. A novel phosphorylation-dependent RNase activity of GAP-SH3 binding protein: a potential link between signal transduction and RNA stability. Mol Cell Biol 1998; 18: 3956-65.

8 Tourriere H, Gallouzi IE, Chebli K, Capony JP, Mouaikel J, van der Geer $\mathrm{P}$, et al. RasGAP-associated endoribonuclease G3Bp: selective RNA degradation and phosphorylation-dependent localization. Mol Cell Biol 2001; 21: 7747-60.

9 Martin S, Zekri L, Metz A, Maurice T, Chebli K, Vignes M, et al. Deficiency of G3BP1, the stress granules assembly factor, results in abnormal synaptic plasticity and calcium homeostasis in neurons. J Neurochem 2013; 125: 175-84.

10 Zekri L, Chebli K, Tourriere H, Nielsen FC, Hansen TV, Rami A, et al. Control of fetal growth and neonatal survival by the RasGAP-associated endoribonuclease G3BP. Mol Cell Biol 2005; 25: 8703-16.
11 Guitard E, Parker F, Millon R, Abecassis J, Tocque B. G3BP is overexpressed in human tumors and promotes $S$ phase entry. Cancer Lett 2001; 162: 213-21.

12 Moutaoufik MT, El Fatimy R, Nassour H, Gareau C, Lang J, Tanguay $\mathrm{RM}$, et al. UVC-induced stress granules in mammalian cells. PLoS One 2014; 9: e112742.

13 Dou N, Chen J, Yu S, Gao Y, Li Y. G3BP1 contributes to tumor metastasis via upregulation of Slug expression in hepatocellular carcinoma. Am J Cancer Res 2016; 6: 2641-50.

14 French J, Stirling R, Walsh M, Kennedy HD. The expression of RasGTPase activating protein SH3 domain-binding proteins, G3BPs, in human breast cancers. Histochem J 2002; 34: 223-31.

15 Aulas A, Caron G, Gkogkas CG, Mohamed NV, Destroismaisons L, Sonenberg N, et al. G3BP1 promotes stress-induced RNA granule interactions to preserve polyadenylated mRNA. J Cell Biol 2015; 209: 73-84.

16 Kedersha N, Panas MD, Achorn CA, Lyons S, Tisdale S, Hickman $\mathrm{T}$, et al. G3BP-Caprin1-USP10 complexes mediate stress granule condensation and associate with 40 S subunits. J Cell Biol 2016; 212 : 845-60.

17 Matsuki H, Takahashi M, Higuchi M, Makokha GN, Oie M, Fujii M. Both G3BP1 and G3BP2 contribute to stress granule formation. Genes Cells 2013; 18: 135-46.

18 Kristensen O. Crystal structure of the G3BP2 NTF2-like domain in complex with a canonical FGDF motif peptide. Biochem Biophys Res Commun 2015; 467: 53-7.

19 Lu J, Sun D, Liu Z, Li M, Hong H, Liu C, et al. SIRT6 suppresses isoproterenol-induced cardiac hypertrophy through activation of autophagy. Transl Res 2016; 172: 96-112 e6.

20 Zhou SG, Zhou SF, Huang HQ, Chen JW, Huang M, Liu PQ. Proteomic analysis of hypertrophied myocardial protein patterns in renovascularly hypertensive and spontaneously hypertensive rats. J Proteome Res 2006; 5: 2901-8.

21 Lu J, Zhang R, Hong H, Yang Z, Sun D, Sun S, et al. The poly(ADPribosyl)ation of FoxO3 mediated by PARP1 participates in isoproterenol-induced cardiac hypertrophy. Biochim Biophys Acta 2016; 1863: 3027-39.

22 Miyoshi T, Nakamura K, Yoshida M, Miura D, Oe H, Akagi S, et al. Effect of vildagliptin, a dipeptidyl peptidase 4 inhibitor, on cardiac hypertrophy induced by chronic beta-adrenergic stimulation in rats. Cardiovasc Diabetol 2014; 13: 43.

23 Mitsuyama S, Takeshita D, Obata K, Zhang GX, Takaki M. Left ventricular mechanical and energetic changes in long-term isoproterenol-induced hypertrophied hearts of SERCA2a transgenic rats. J Mol Cell Cardiol 2013; 59: 95-106.

24 Divakaran VG, Evans S, Topkara VK, Diwan A, Burchfield J, Gao F, et al. Tumor necrosis factor receptor-associated factor 2 signaling provokes adverse cardiac remodeling in the adult mammalian heart. Circ Heart Fail 2013; 6: 535-43.

25 Gupta S, Young D, Maitra RK, Gupta A, Popovic ZB, Yong SL, et al. Prevention of cardiac hypertrophy and heart failure by silencing of NFkappaB. J Mol Biol 2008; 375: 637-49.

26 Abdelhamid G, El-Kadi AO. Buthionine sulfoximine, an inhibitor of glutathione biosynthesis, induces expression of soluble epoxide hydrolase and markers of cellular hypertrophy in a rat cardiomyoblast cell line: roles of the NF-kappaB and MAPK signaling pathways. Free Radic Biol Med 2015; 82: 1-12.

27 Chen J, Chen Y, Zhu W, Han Y, Han B, Xu R, et al. Specific LPA receptor subtype mediation of LPA-induced hypertrophy of cardiac myocytes and involvement of Akt and NFkappaB signal pathways. J Cell Biochem 2008; 103: 1718-31. 
28 Prigent M, Barlat I, Langen H, Dargemont C. IkappaBalpha and IkappaBalpha/NF-kappa B complexes are retained in the cytoplasm through interaction with a novel partner, RasGAP SH3-binding protein 2. J Biol Chem 2000; 275: 36441-9.

29 Lypowy J, Chen IY, Abdellatif M. An alliance between Ras GTPaseactivating protein, filamin C, and Ras GTPase-activating protein SH3 domain-binding protein regulates myocyte growth. J Biol Chem 2005; 280: 25717-28.

30 He M, Yang Z, Abdellatif M, Sayed D. GTPase activating protein (Sh3 Domain) binding protein 1 regulates the processing of microRNA-1 during cardiac hypertrophy. PLoS One 2015; 10: e0145112.

31 Kennedy D, French J, Guitard E, Ru K, Tocque B, Mattick J. Characterization of G3BPs: tissue specific expression, chromosomal localisation and rasGAP(120) binding studies. J Cell Biochem 2001; 84: 173-87.

32 Katoh M, Katoh M. Transcriptional mechanisms of WNT5A based on NF-kappaB, Hedgehog, TGFbeta, and Notch signaling cascades. Int J Mol Med 2009; 23: 763-9.

33 Bikkavilli RK, Malbon CC. Wnt3a-stimulated LRP6 phosphorylation is dependent upon arginine methylation of G3BP2. J Cell Sci 2012; 125 : 2446-56.

34 Kaye D, Esler M. Sympathetic neuronal regulation of the heart in aging and heart failure. Cardiovasc Res 2005; 66: 256-64.

35 Barnes CJ, Li F, Mandal M, Yang Z, Sahin AA, Kumar R. Heregulin induces expression, ATPase activity, and nuclear localization of G3BP, a Ras signaling component, in human breast tumors. Cancer Res 2002; 62: 1251-5.

36 Shi M, Liu D, Duan H, Qian L, Wang L, Niu L, et al. The beta2-adrenergic receptor and Her2 comprise a positive feedback loop in human breast cancer cells. Breast Cancer Res Treat 2011; 125: 351-62.

37 Planavila A, Iglesias R, Giralt M, Villarroya F. Sirt1 acts in association with PPARalpha to protect the heart from hypertrophy, metabolic dysregulation, and inflammation. Cardiovasc Res 2011; 90: 276-84.

38 Zou J, Li H, Chen X, Zeng S, Ye J, Zhou C, et al. C/EBPbeta knockdown protects cardiomyocytes from hypertrophy via inhibition of p65NFkappaB. Mol Cell Endocrinol 2014; 390: 18-25.

39 Freund C, Schmidt-Ullrich R, Baurand A, Dunger S, Schneider W, Loser $\mathrm{P}$, et al. Requirement of nuclear factor-kappaB in angiotensin II- and isoproterenol-induced cardiac hypertrophy in vivo. Circulation 2005; 111: 2319-25.

40 Li YQ, Zhang ZX, Xu YJ, Ni W, Chen SX, Yang Z, et al. N-Acetyl-Lcysteine and pyrrolidine dithiocarbamate inhibited nuclear factorkappaB activation in alveolar macrophages by different mechanisms. Acta Pharmacol Sin 2006; 27: 339-46.

41 Wang Z, Wu W, Tang M, Zhou Y, Wang L, Xu W, et al. NF-kappaB pathway mediates vascular smooth muscle response to nicotine. Int J Biochem Cell Biol 2013; 45: 375-83.

42 Moellering D, McAndrew J, Jo H, Darley-Usmar VM. Effects of pyrrolidine dithiocarbamate on endothelial cells: protection against oxidative stress. Free Radic Biol Med 1999; 26: 1138-45.

43 Yokoo T, Kitamura M. Antioxidant PDTC induces stromelysin expression in mesangial cells via a tyrosine kinase-AP-1 pathway. Am J Physiol 1996; 270: F806-11.

44 Bakkar N, Guttridge DC. NF-kappaB signaling: a tale of two pathways in skeletal myogenesis. Physiol Rev 2010; 90: 495-511.

45 Latimer M, Ernst MK, Dunn LL, Drutskaya M, Rice NR. The N-terminal domain of IkappaB alpha masks the nuclear localization signal(s) of p50 and c-Rel homodimers. Mol Cell Biol 1998; 18: 2640-9.

46 Huguet C, Crepieux P, Laudet V. Rel/NF-kappa B transcription factors and I kappa B inhibitors: evolution from a unique common ancestor. Oncogene 1997; 15: 2965-74.

47 Weber KT, Anversa P, Armstrong PW, Brilla CG, Burnett JC $\mathrm{Jr}$, Cruickshank JM, et al. Remodeling and reparation of the cardiovascular system. J Am Coll Cardiol 1992; 20: 3-16. 